\title{
El Programa de Reforzamiento Comunitario más Terapia de Incentivo para el tratamiento de la adicción a la cocaína
}

\author{
Roberto Secades-Villa'; Olaya García-Rodríguez'; Helí Álvarez Rodríguez²; Arcadio Río Rodríguez²; \\ José R. Fernández-Hermida ${ }^{1}$; José Luis Carballo ${ }^{1}$
}

(1) Departamento de Psicología. Universidad de Oviedo.

(2) Proyecto Hombre. Asturias.

Enviar correspondencia a:

Roberto Secades-Villa. Facultad de Psicología. Universidad de Oviedo. Plaza Feijoo s/n 33003 Oviedo - Spain. e-mail: secades@uniovi.es

Recibido: Mayo 2006.

Aceptado: Octubre 2006

\section{RESUMEN}

El consumo de cocaína es un problema de tendencia creciente en nuestro país. La ausencia de fármacos eficaces para el tratamiento de la adicción a esta sustancia así como de programas terapéuticos validados empíricamente hace necesario acudir a programas que han demostrado su eficacia en otros países. El objetivo de este estudio es evaluar la eficacia de uno de los programas con mejores resultados en Estados Unidos: La Aproximación de Reforzamiento Comunitario (CRA) más Terapia de Incentivo para la adicción a la cocaína. Se pretenden valorar las tasas de retención y abandono en el tratamiento, y el consumo de cocaína durante los tres primeros meses de intervención. La muestra estuvo compuesta por 37 pacientes adictos a la cocaína que fueron asignados aleatoriamente a dos condiciones: experimental (CRA más Terapia de Incentivo) y control (Tratamiento Estándar), ambos en un contexto asistencial ambulatorio. Los resultados mostraron que el $85,7 \%$ de los pacientes del grupo experimental completó 12 semanas de tratamiento frente al 69,6\% del grupo control. En el grupo experimental, el $57,1 \%$ de los pacientes se mantuvo abstinente de forma continuada frente al 39,1\% del grupo control. Estos resultados coinciden con los encontrados en estudios previos realizados siempre fuera de España. No obstante, se necesitan estudios a más largo plazo y con un mayor número de sujetos para confirmar la eficacia de este programa.

Palabras clave: dependencia a la cocaína, CRA, terapia de incentivo, retención, reforzamiento comunitario.

\begin{abstract}
Cocaine use is an increasingly serious problem in Spain. The absence of effective drugs for the treatment of cocaine addiction and of empirically validated therapy programmes makes it necessary to resort to programmes that have shown their effectiveness in other countries. The aim of the present study was to explore the effectiveness of one of the programmes that has obtained the best results in the United States: the Community Reinforcement Approach (CRA) Plus Vouchers for the treatment of cocaine addiction. We assessed treatment retention and dropout rates and cocaine use during the first three months of intervention. The sample was made up of 37 patients addicted to cocaine who were assigned at random to one of two conditions: experimental (CRA Plus Vouchers) or control (Standard Treatment), both in the outpatient context. The results showed that $85.7 \%$ of the experimental group patients completed 12 weeks of treatment, compared to $69.6 \%$ of the control group. In the experimental group, $57.1 \%$ of the patients maintained continuous abstinence, compared to $39.1 \%$ of the control group. These results coincide with those of previous studies, all from outside Spain. Nevertheless, longer-term studies with larger samples are necessary in order to confirm the effectiveness of this program.
\end{abstract}

Key words: Cocaine dependence, CRA, vouchers, retention, community reinforcement.

\section{INTRODUCCIÓN}

egún la última Encuesta Escolar del Plan Nacional sobre Drogas (Plan Nacional sobre Drogas, 2005), el consumo habitual de cocaína ha pasado del $1,1 \%$ al 3,8\% entre la población escolar española en los últimos 10 años. De acuerdo con el European Monitoring Centre for Drugs and Drug Addiction (EMCDDA), España, junto con Gran Bretaña, es el país con las tasas de consumo de cocaína más altas. Al mismo tiempo, la demanda de trata- 
miento por abuso de cocaína se ha incrementado de forma muy importante en nuestro país durante los últimos años (Aguilar de Arcos, Verdejo García, Sánchez Barrera, López Jiménez y Pérez García, 2005; García Rodríguez et al., 2005). De hecho, los países con mayor porcentaje de demandas de tratamiento en Europa son España y Holanda (EMCDDA, 2005). Estos datos han sido confirmados en el último informe de Naciones Unidas, en donde se muestra que España es el segundo país con las tasas más altas de abuso de cocaína, solo después de Estados Unidos (UNODC, 2005).

A pesar de todos estos indicadores que alertan del incremento del consumo de cocaína, los recursos asistenciales y los protocolos de tratamiento para la adicción a esta sustancia no han tenido en nuestro país el mismo desarrollo que aquellos dirigidos a la dependencia a los opiáceos. A esto hay que añadir que, como ocurre con otras sustancias, como por ejemplo, la marihuana (Ramos, 2006), todavía no se ha encontrado ninguna medicación eficaz que sea considerada como un tratamiento de primera elección para esta adicción (San Molina y Arranz, 2001; Solé Puig, 2001; van den Brink y van Ree, 2003).

Por otra parte, los programas de manejo de contingencias han tenido un fuerte impulso en los últimos años y, en particular, el Programa de Reforzamiento Comunitario más terapia de incentivo (CRA + Vouchers) para el tratamiento de la adicción a la cocaína ha demostrado una alta eficacia en estudios bien controlados. Por este motivo, se trata de un programa que ha sido avalado por el National Institute on Drug Abuse (NIDA) de Estados Unidos.

El CRA + terapia de incentivo integra el programa del Reforzamiento Comunitario, originalmente desarrollado como un tratamiento efectivo para el alcoholismo (Hunt y Azrin, 1973) con un programa de manejo de contingencias, en donde los pacientes pueden ganar puntos a cambio de mantenerse en el tratamiento sin consumir cocaína. Estros puntos son canjeables por determinados reforzadores que contribuyen a alcanzar los objetivos del programa.

El grupo de Higgins de la Universidad de Vermont ha llevado a cabo varios ensayos clínicos examinando la eficacia de este programa. En dos de estos trabajos (Higgins et al., 1991, 1993), el CRA + terapia de incentivo se mostró superior a un programa de consejo psicológico tradicional varios meses después del tratamiento. En un tercer ensayo (Higgins et al., 1994), se comprobó la eficacia diferencial del componente de incentivos. Para ello, los pacientes fueron asignados al azar a un grupo que recibía el tratamiento completo o CRA solo. Los resultados mostraron que los sujetos que recibieron el programa completo alcanzaron tasas de abstinencia significativamente superiores que los sujetos del grupo CRA solo. Otro estudio similar (Hig- gins et al., 2003), comprobó la eficacia del otro componente (CRA). Para ello, los pacientes fueron asignados al azar a dos grupos; un grupo recibía el tratamiento completo y otro grupo sólo recibía incentivos. Los resultados fueron similares a los del estudio anterior, comprobándose así que ambos componentes son necesarios para una mayor eficacia del programa.

En un estudio reciente, Higgins, et al. (2000) compararon un grupo de CRA más terapia de incentivo contingente a la abstinencia, con un grupo de CRA más incentivos independientes de los resultados de los análisis de orina. Los resultados al año de seguimiento mostraron diferencias significativas a favor del "grupo contingente".

Por otra parte, han sido llevados a cabo varios ensayos clínicos con pacientes que abusaban de la cocaína mientras estaban a tratamiento con sustitutivos por dependencia a opiáceos. En todos estos estudios, la terapia de incentivos incrementó significativamente la abstinencia de la cocaína (por ejemplo, Bickel, Amass, Higgins, Badger y Esch, 1997; Silverman et al., 1996; Silverman et al., 1998; Stitzer, Iguchi y Felch, 1992). También se ha adaptado esta terapia a poblaciones especiales mostrando excelentes resultados. En concreto, con pacientes esquizofrénicos adictos a la cocaína (Shaner et al., 1997), y con mujeres embarazadas (Higgins, Clough y Wallerstedt, 1995).

En una revisión de la literatura científica, once de los trece estudios publicados (85\%), encontraron que el uso de incentivos mostraba efectos positivos en el seguimiento a largo plazo de los sujetos (Higgins, Heil y Lussier, 2004).

El uso de incentivos también se ha extendido al tratamiento del abuso de otras sustancias (nicotina, alcohol, cannabis y opiáceos) (Higgins, Alessi y Dantona, 2002). La gran mayoría de estos estudios informan de mejoras significativas en el uso de drogas y en otras conductas asociadas (Higgins, Heil y Lussier, 2004).

En definitiva, los resultados de los estudios demuestran que los programas de incentivos basados en el manejo de contingencias son tratamientos eficaces para el tratamiento de la adicción a diferentes sustancias y con poblaciones diferentes (Higgins, Heil y Lussier, 2004; Roozen et al. 2004; Secades Villa y Fernández Hermida, 2003).

No obstante, se debe resaltar el hecho de que todos los estudios clínicos exitosos que se han publicado hasta la fecha han sido llevados a cabo en Estados Unidos, por lo que sería de gran interés analizar el funcionamiento de ese programa en una realidad social y cultural diferente a la de ese país. Por otra parte, la mayoría de estos trabajos han sido llevados a cabo en contextos de investigación muy controlados, por lo que son necesarios ensayos clínicos en donde 
se analice la efectividad de este modelo de intervención en contextos comunitarios.

En este trabajo se presentan los primeros resultados de una investigación que trata de cubrir esta necesidad. En particular, los objetivos de este estudio son dos: en primer lugar, analizar la eficacia del programa CRA más terapia de incentivo en comparación con un tratamiento tradicional para la adicción a la cocaína durante los tres meses de tratamiento y, en segundo, valorar su aplicabilidad en un contexto comunitario en España.

\section{MÉTODOS}

\section{Participantes}

La muestra del estudio fue extraída de forma aleatoria, del total de pacientes que acudieron a una clínica de Proyecto Hombre en Asturias por sus problemas con la cocaína.
Los criterios de inclusión fueron los siguientes: cumplir los criterios diagnósticos para la dependencia a la cocaína según el DSM-IV (APA, 2002), ser mayor de veinte años, y residir en el centro de la región. Sufrir trastornos psicopatológicos graves (por ejemplo, psicosis o demencia) se tomó como criterio de exclusión.

El total de pacientes seleccionados fue de 37, (83,8\% hombres y $16,2 \%$ mujeres). La edad media de los participantes fue de 28,5 años (rango 20 - 40).

La asignación de los pacientes al grupo control (programa tradicional) o al grupo experimental (CRA más terapia de incentivo) se hizo de forma aleatoria. Para ello, se utilizó un listado de números aleatorios generados por ordenador. El número de pacientes asignados a los dos grupos fue de 14 en el grupo experimental y 23 en el grupo control. La razón por la que se asignaron más participantes al grupo control que al experimental fue la alta tasa de abandonos del primer grupo.

En la Tabla 1 se presentan algunas características de los pacientes que participaron en el estudio. No

Tabla 1. Características de los participantes

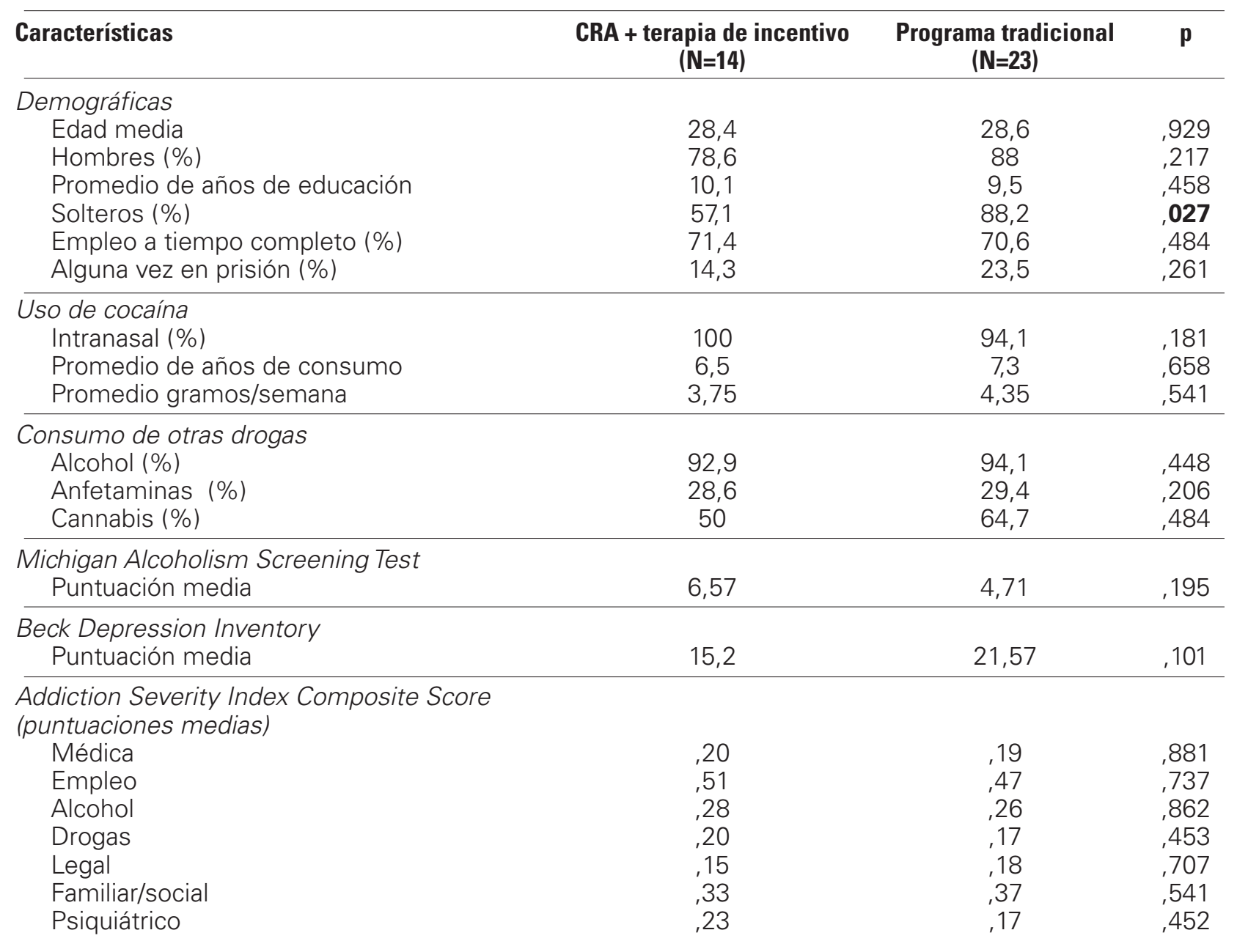


existían diferencias estadísticamente significativas en ninguna de las variables medidas en la línea base, excepto en el porcentaje de solteros, que era superior en el grupo control.

\section{Instrumentos}

Durante la admisión, que duró aproximadamente tres sesiones de una hora, se les administraron a los pacientes los siguientes instrumentos: Criterios de dependencia a la cocaína DSM-IV-TR (American Psychiatric Association, 2002), Michigan Alcoholism Screening Test (MAST) (Selzer, 1971), Beck Depression Inventory (BDI) (Beck, Ward, Mendelson, Mock y Erbaugh, 1961) y EuropASI (Kokkevi y Hartgers, 1995). Además de estos instrumentos, los pacientes se sometieron a un reconocimiento médico completo.

Para la detección de cocaína en la orina se utilizarón los reactivos Quickscreen Test de Eve Layper. Estos reactivos detectan el consumo de cocaína de los pacientes a través de la orina de forma rápida. Los niveles de corte que detectan estos test son los recomendados por el Substance Abuse and Mental Health Services Administration (SAMHSA).

\section{Procedimiento}

\section{CRA más terapia de incentivo}

El programa CRA más Terapia de Incentivo es un programa ambulatorio con una duración de 12 meses que tiene como objetivo fundamental la abstinencia a la cocaína. Los componentes de la terapia son seis: terapia de incentivo, habilidades de afrontamiento de consumo de drogas, cambios en el estilo de vida, asesoramiento en las relaciones de pareja, consumo de otras drogas y tratamiento de otros trastornos.

El protocolo se aplicó de acuerdo a la versión original de Budney y Higgins (1998), con una sola diferencia, y es que en nuestro caso, la aplicación de la mayor parte de los módulos se hizo de forma grupal, debido a la necesidad de adaptar los protocolos del programa a las características del centro en donde se llevó a cabo la intervención. Los pacientes acudían a dos sesiones grupales semanales y si era necesario, se llevaban a cabo sesiones individuales para resolver problemas puntuales. El asesoramiento en relaciones de pareja, algunos componentes de cambios en el estilo de vida y el tratamiento de otros trastornos asociados se trabajan siempre de forma individual.

El sub-componente de la terapia de incentivo es un procedimiento de manejo de contingencias mediante el que se refuerza sistemáticamente la abstinencia a la cocaína. A cambio de resultados negativos en los análisis de orina se ganan bonos que son canjeados por determinados incentivos (bienes o servicios) que ayudan a los pacientes a alcanzar los objetivos terapéuticos y a mejorar el estilo de vida (actividades de tiempo libre, transporte, cursos de formación etc.). El programa de incentivos fue financiado principalmente a través de donaciones de empresas y organismos públicos. Los incentivos que se utilizaron fueron: bonos canjeables por actividades de ocio y tiempo libre (entradas para cines, teatros, museos, eventos deportivos, gimnasios, deportes de aventura, etc.), tarjetas regalo o bonos descuento para grandes superficies y pequeños comercios, menús gratuitos en restaurantes, servicios de peluquería y belleza, suscriciones gratuitas a periódicos regionales, cursos de formación (idiomas, informática, etc.) y transporte público gratuito. En ningún caso se utilizó dinero como método de reforzamiento de la abstinencia. Los análisis de orina se realizan tres veces por semana durante los primeros tres meses del programa, dos por semana en los siguientes tres meses y de forma aleatoria en los últimos seis meses. Todas las muestras negativas recogidas en los tres primeros meses son reforzadas con incentivos. Durante los meses tres a seis, se introduce un sistema aleatorio con el que sólo se refuerzan la mitad de las muestras negativas.

El incremento en el valor de cada analítica negativa consecutiva se hizo también de acuerdo a lo establecido en el manual original del programa.

\section{Programa tradicional de Proyecto Hombre}

Se trata de un programa ambulatorio, libre de drogas, de corte cognitivo conductual. La duración del programa es de 18 meses. El programa se estructura en tres fases de diferente duración cada una de ellas. Los pacientes acuden dos veces por semana a sesiones grupales de una hora y media de duración aproximadamente y, si es necesario, se acuerdan con el terapeuta sesiones individuales para resolver problemas puntuales. Los componentes de las sesiones de grupo son fundamentalmente: información sobre drogas, incremento de la conciencia del problema de la adicción, expresión de emociones, solución de problemas y prevención de recaídas. Además de los componentes propios de las sesiones grupales, se cuenta con otros componentes como, grupos psicoeducativos sobre salud y drogas, talleres formativo-laborales y grupos de apoyo para las familias de los pacientes.

Los análisis de orina se realizan los mismos días que los pacientes acuden a grupo. Los pacientes de este grupo no recibían ningún tipo de incentivo a cambio de mantenerse abstinentes.

\section{Análisis de datos}

Se llevaron a cabo diferentes análisis descriptivos y de frecuencias para describir las características de los participantes. Se realizaron análisis bivariados (prueba 
de significación estadística de diferencias entre proporciones con z como estadístico de contraste para las variables dicotómicas y t de Student para las variables continuas) para comparar a los sujetos de los dos grupos de tratamiento en la línea base y para analizar las diferencias en el consumo de cocaína y en la retención en el tratamiento.

El nivel de confianza fue del $95 \%$ y el paquete estadístico utilizado el SPSS-11.

\section{RESULTADOS}

\section{Tasas de retención}

El 85,7\% de los pacientes del grupo experimental (CRA más terapia de incentivo) completó 3 meses de tratamiento frente al 69,6\% de pacientes del grupo control (programa de tratamiento tradicional) $(p=.13)$. Entre los que no siguieron en el tratamiento, sólo uno de los pacientes del grupo experimental $(17,1 \%)$ fue abandono, frente al 30,4\% de los sujetos del grupo control $(p=.04)$.

La desviación que se aprecia en el grupo experimental entre el porcentaje de retención y abandono se explica porque uno de los pacientes del grupo experimental fue derivado a un programa residencial a los dos meses de tratamiento, por lo que no ha sido contabilizado como retenido pero tampoco ha sido clasificado como abandono.

\section{Abstinencia a la cocaína}

En el Gráfico 1 se puede ver el porcentaje de pacientes de cada uno de los grupos que ha conseguido mantenerse abstinente de forma continuada durante las doce primeras semanas de tratamiento. El grupo CRA más terapia de incentivo supera sistemáticamente al tratamiento convencional en el porcentaje de pacientes que consiguen abstinencia continuada durante los tres primeros meses de tratamiento.
Como se puede apreciar, a las cuatro semanas de tratamiento, el $85 \%$ de los pacientes del grupo experimental se habían mantenido abstinentes de forma continuada frente al $70,6 \%$ de los sujetos del grupo control $(p=.38)$. A las ocho semanas, el 71,4\% del grupo experimental frente al $45,5 \%$ del grupo control $(p=.06)$, y a las doce semanas, el 57,1\% del grupo experimental frente al 39,1\% del grupo control $(p=.15)$.

Si tenemos en cuenta el número máximo de semanas que los pacientes han conseguido mantenerse abstinentes durante los seis meses de tratamiento, sin considerar que el inicio de esta abstinencia deba coincidir con el inicio del tratamiento, de nuevo los pacientes del grupo experimental superan a los pacientes del grupo control. La duración media de la abstinencia fue de 10,5 semanas en el grupo experimental frente a 7,86 en el grupo control $(p=.01)$. Estos datos pueden verse en el Gráfico 2.

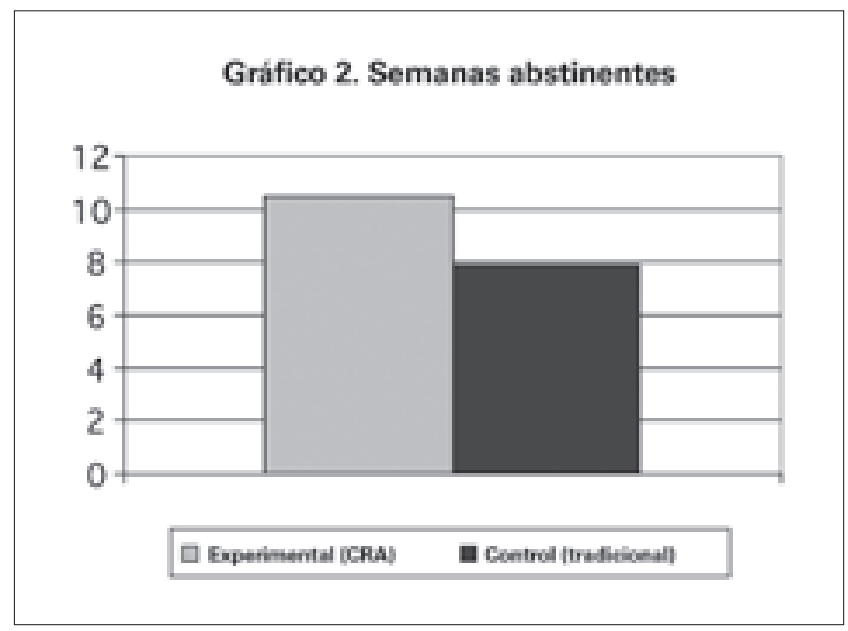

En el Gráfico 3 se puede observar el porcentaje de analíticas positivas para el consumo de cocaína (detección de benzoilecgonina en la orina) del total de las realizadas en ambos grupos. El porcentaje de de analíticas positivas en el grupo experimental fue del $1,83 \%$ frente al $10,25 \%$ en el grupo control $(p=.02)$.

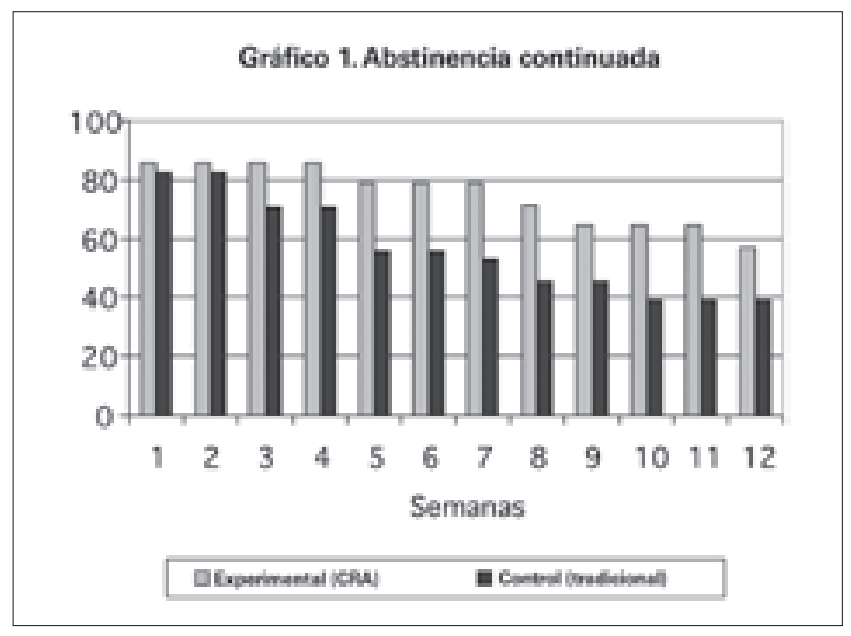

Gráfico 3.Analiticas positivas de consumo de cocaina

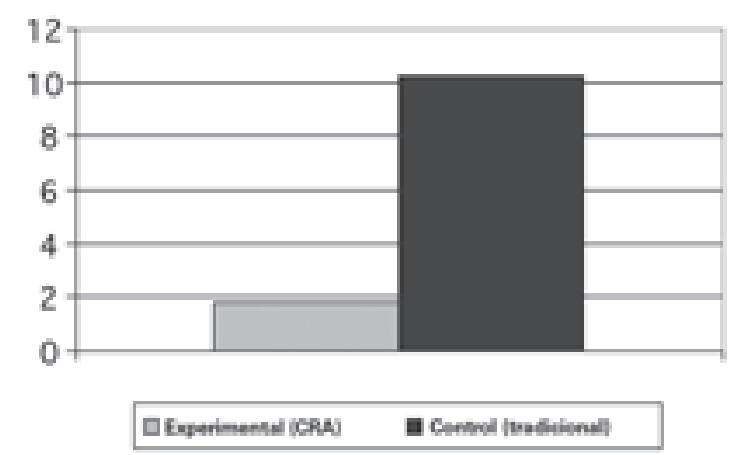




\section{DISCUSIÓN}

El objetivo principal de este estudio fue analizar la eficacia inicial del programa CRA más terapia de incentivo en comparación con un programa estándar, para el tratamiento de la adicción a la cocaína.

Los datos muestran que el grupo experimental obtiene mejores resultados que el grupo comparativo. Así, las tasas de retención a los tres meses de tratamiento fueron mejores entre los pacientes del grupo CRA más terapia de incentivo que entre los pacientes del grupo control. El consumo de cocaína (medido en términos de tasas de abstinencia continuada y de porcentaje de analíticas positivas) fue significativamente inferior en el grupo experimental en comparación con el grupo control.

No obstante, el número reducido de sujetos provoca que, en algunos casos, estas diferencias no alcancen significatividad estadística. A pesar de esto, los datos muestran una tendencia evidente de menor consumo y mayor retención en el grupo experimental en comparación con el grupo control. Este es un dato relevante, ya que los resultados de múltiples estudios muestran cómo la retención y la abstinencia durante el tratamiento son predictores muy importantes de la abstinencia a largo plazo (Higgins, Badger y Budney, 2000).

Por otra parte, cuando el programa CRA más terapia de incentivo ha sido comparado con otros tratamientos, lo ha hecho con el modelo de los doce pasos (por ejemplo, Higgins et al., 1991). En nuestro caso, hemos comparado CRA más terapia de incentivo con un tratamiento, en gran medida, cognitivo-conductual. Este hecho también puede explicar, en parte, porqué en estudios previos se obtienen diferencias mayores entre el grupo experimental y el grupo control.

Una barrera habitualmente esgrimida para la aplicabilidad y diseminación de este tipo de intervenciones es su relativo alto coste. En nuestro caso, pensamos que una forma alternativa de reducir los costes del programa CRA es aplicar algunos de sus componentes en formato de grupo. A la vista de los datos obtenidos, no parece que el formato grupal disminuya la eficacia del programa CRA más terapia de incentivo. Las tasas de retención y consumo son muy similares a los de estudios en los que la aplicación se ha hecho de forma individual (eg., Higgins et al., 1993; Higgins et al., 1994).

Como conclusión, se puede afirmar que el programa CRA más terapia de incentivo puede ser una estrategia efectiva y viable en contextos naturales y que este modelo puede ser generalizable fuera de Estados Unidos, ya que los resultados iniciales muestran unas tasas de eficacia similares a las encontradas en los estudios originales. No obstante, estos resultados han de verse confirmados en estudios de seguimiento más largos y con mayor número de sujetos.

\section{AGRADECIMIENTOS}

Este proyecto ha sido financiado por la Delegación del Gobierno para el Plan Nacional de Drogas (Ref. MINT-03-01) así como por una beca predoctoral de la Universidad de Oviedo (Ref. UNIOVI-04-BECDOC05) y una beca predoctoral de la Fundación para el Fomento de la Investigación Científica Aplicada y la Tecnología (FICYT) de Asturias (Ref. BP05-002). Los autores desean agradecer también la colaboración de los responsables de la Fundación Proyecto Hombre de Asturias.

\section{REFERENCIAS}

Aguilar de Arcos, F., Verdejo García, A., Sánchez Barrera, M. B., López Jiménez, A. y Pérez García, M. (2005). Perfil emocional de los consumidores de "revuelto" en comparación con consumidores de heroína y de cocaína. Adicciones, 17(2), 131-138.

American Psychiatric Association (2002). DSM-IV-TR. Manual diagnóstico y estadístico de los trastornos mentales. Barcelona: Masson.

Beck, A. T., Ward, C. H., Mendelson, M., Mock, J. y Erbaugh, J. (1961). An inventory for measuring depression. Archives of General Psychiatry, 4, 561-571.

Bickel, W. K., Amass, L., Higgins, S. T., Badger, G. J. y Esch, R. A. (1997). Effects of adding behavioral treatment to opioid detoxification with buprenorphine. Journal of Consulting and Clinical Psychology, 65(5), 803-810.

Budney, A. J. y Higgins, S. T. (1998). A Community Reinforcement Approach: Treating Cocaine Addiction. Retrieved February 7, 2006, from http://www. drugabuse.gov/TXManuals/CRA/CRA1.html

European Monitoring Centre for Drugs and Drug Addiction (2005). The state of the drugs problem in Europe. Luxembourg: EMCDDA

García Rodríguez, O., Secades Villa, R., Fernández Hermida, J. R., Carballo Crespo, J. L., Errasti Pérez, J. M. y Alhalabí Díaz, S. (2005). Comparación de pacientes cocainómanos y heroinómanos en el EuropASI. Adicciones, 17(1), 33-42.

Higgins, S. T., Alessi, S. M., y Dantona, R. L. (2002). Voucherbased incentives. A substance abuse treatment innovation. Addictive Behaviors, 27(6), 887-910.

Higgins, S.T., Badger, G.J. y Budney, A.J. (2000). Initial abstinence and success in achieving longer term cocaine abstinence. Experimental and Clinical Psychopharmacology, 8, 377-386.

Higgins, S. T., Budney, A. J., Bickel, W. K., Foerg, F. E., Donham, R. y Badger, G. J. (1994). Incentives improve outcome in outpatient behavioral treatment of cocaine dependence. Archives of General Psychiatry, 51(7), 568-576.

Higgins, S. T., Budney, A. J., Bickel, W. K., Hughes, J. R., Foerg, F. y Badger, G. (1993). Achieving cocaine abstinence with a behavioral approach. American Journal of Psychiatry, 150(5), 763-769. 
Higgins, P. G., Clough, D. H. y Wallerstedt, C. (1995). Drugtaking behaviours of pregnant substance abusers in treatment. Journal of Advanced Nursing, 22(3), 425432.

Higgins, S. T., Delaney, D. D., Budney, A. J., Bickel, W. K., Hughes, J. R., Foerg, F. y Fenwick, J. W. (1991). A behavioral approach to achieving initial cocaine abstinence. American Journal of Psychiatry, 148(9), 1218-1224.

Higgins, S. T., Heil, S. H. y Lussier, J. P. (2004). Clinical implications of reinforcement as a determinant of substance use disorders. Annual Review of Psychology, $55,431-461$.

Higgins, S. T., Sigmon, S. C., Wong, C. J., Heil, S. H., Badger, G. J., Donham, R., Dantona, R. L. y Anthony, S. (2003). Community reinforcement therapy for cocainedependent outpatients. Archives of General Psychiatry, 60(10), 1043-1052.

Hunt, G. M., y Azrin, N. H. (1973). A communityreinforcement approach to alcoholism. Behaviour Research and Therapy, 11(1), 91-104.

Kokkevi, A. y Hartgers, C. (1995). European adaptation of a multidimensional assessment instrument for drug and alcohol dependence. European Addiction Research, 1, 208-210.

Plan Nacional sobre Drogas (2005). Encuesta estatal sobre uso de drogas en enseñanzas secundarias 2004. Madrid: Delegación del Gobierno para el Plan Nacional sobre Drogas.

Ramos Atance, J.A. (2006). Búsqueda de un tratamiento farmacológico para la dependencia a la marihuana. Adicciones, $18(1), 5-10$.

Roozen, H. G., Boulogne, J. J., van Tulder, M. W., van den Brink, W., De Jong, C. A., y Kerkhof, A. J. (2004). A systematic review of the effectiveness of the community reinforcement approach in alcohol, cocaine and opioid addiction. Drug Alcohol Depend, 74(1), 1-13.

San Molina, L. y Arranz, B. (2001). Aproximación terapéutica de la dependencia de cocaína. Adicciones, 13(Suplemento 2), 191-208.
Secades Villa, R. y Fernández Hermida, J. R. (2003). Guía de tratamientos psicológicos eficaces en la drogadicción: alcoholismo, cocaína y heroína. En M. Pérez Alvarez, J. R. Fernández Hermida, C. Fernández Rodríguez y I. Amigo Vázquez (Eds.), Guía de tratamientos psicológicos eficaces. I. Adultos (pp. 107-139). Madrid: Ediciones Pirámide.

Selzer, M. L. (1971). The Michigan alcoholism screening test: the quest for a new diagnostic instrument. The American Journal of Psychiatry, 127, 1653-1658

Shaner, A., Roberts, L.J., Eckman, T.A., Tucker, D.E., Tsuang, J.W., Wilkins, J.N. y Mintz, J. (1997). Monetary reinforcement of abstinence from cocaine among mentally ill patients with cocaine dependence. Psychiatric Services, 48, 807-810.

Silverman, K., Wong, C.J., Higgins, S.T., Brooner, R.K., Montoya, I.D., Contoreggi,C., Umbricht-Schneiter, A., Schuster, C.R. y Preston, K.L. (1996). Increasing opiate abstinence through voucher-based reinforcement therapy. Drug and Alcohol Dependence, 41, 157-65.

Silverman, K., Wong, C.J., Umbricht-Schneiter, A., Montoya, I.D., Schuster, C.R. y Preston, K.L. (1998). Broad beneficial effects of cocaine abstinence reinforcement among methadone patients. Journal of Consulting and Clinical Psychology, 66, 811-824.

Solé Puig, J. (2001). Tratamiento del consumo de cocaína. Integrando psicoterapia y farmacoterapia. Adicciones, 13(Suplemento 2), 209-225.

Stitzer, M.L., Iguchi, M.Y. y Felch, L.J. (1992). Contingent take-home incentive: effects on drug use of methadonemaintenance patients. Journal of Consulting and Clinical Psychology, 60, 927-934.

United Nations Office for Drugs and Crime (2005). World Drug Report 2005. Retrieved April 28, 2005, from www.unodc.org

Van den Brink, W. y van Ree, J. M. (2003). Pharmacological treatments for heroin and cocaine addiction. European Neuropsychopharmacology, 13(6), 476-487. 
